\title{
Recent Developments in Acoustics and Vibration
}

\author{
Malcolm J. Crocker \\ Department of Mechanical Engineering Aubum University, AL 36849, USA
}

\begin{abstract}
In recent years there have been rapid advances in digital computers, the miniaturization of electronic circuits and the development of new materials. In the acoustics and vibration fields these advances have led to a continual increase in computational power and speed, improved acoustics and vibration transducers and instrumentation and better measurement techniques. In many cases the developments have been synergistic; new experimental knowledge has led to improved theoretical models and approaches and vice versa. Improved computers have allowed the development of a host of computer programs and increasing numbers have become available as commercial acoustics and vibration software. Of particular importance has been the development of numerical calculation schemes such as the finite element method (FEM) and the boundary element method (BEM) which have led to much improved predictive capabilities in many fields. However, advances have been in many other areas of acoustics as well and not confined just to such numerical prediction schemes. As examples, a few of these advances will be concisely summarized including: increased knowledge of and use of FEM and BEM, Computational Aeroacoustics, Sonochemistry, Thermoacoustic Engines, Active Noise and Vibration Control, Sound Intensity Measurements and their uses, Techniques of Speech Coding and Recognition of Speech, Ultrasonics in Medical Diagnostics, and Cochlear Mechanics.
\end{abstract}

\section{INTRODUCTION}

The development of the digital computer in the 1950's signaled the beginning of increasingly rapid developments in science. Even the early digital computers enabled rapid calculations and analytical solutions of problems to be made that were previously insoluble because of the enormous amount of calculations and thus time and manpower needed for their solution. Further developments in digital computers have continued this trend enabling ever more rapid and massive calculations and solutions of problems of increasing complexity. The developments have also brought lower costs and miniaturization of components and the late 1970's also saw the development of the desktop and portable computers that put computing power into the hands of increasing numbers of scientists. These trends have continued unabated to the present day. In fact the established trend may even accelerate. Moore's law which for the last 30 years has predicted very accurately that the amount of memory that could be stored on a computer chip (and thus its speed and efficiency) doubled every 18 months recently seems to be violated. On 17 September 1997, Intel announced a new chip that uses multilevel cell flash memory. In less than a week on 21 September 1997, I.B.M. announced that it had solved a fundamental problem holding back the development of faster semiconductor chips and that early in 1998 it will begin manufacturing chips that are smaller and up to $40 \%$ faster than the most advanced chips currently being produced commercially. Such rapid advances in computer technology may lead to a doubling of chip performance every nine instead of eighteen months as predicted by Moore's law. Such rapid developments in computer power (and the attendant reduction in cost) have had dramatic effects on the solution of acoustics and vibration problems. Some very visible results have been the development of the finite element method (FEM) and the boundary element method (BEM) that can solve complicated acoustics and vibration problems and of increasing numbers of software programs that can run on mainframe and more and more on desktop and portable computers. Digital computers have also increasingly been built into instrumentation and analysis equipment enabling more sophisticated experimentation in acoustics and vibration and more rapid and sophisticated analysis of the results. The development of piezoelectric materials has also led to advances in the design of acoustics and vibration transducers. All these and other developments have had a synergistic effect and have enabled or aided advances in several fields of acoustics and vibration. Some of these are outlined in the following.

\section{NUMERICAL APPROACHES: FINITE ELEMENTS AND BOUNDARY ELEMENTS}

In cases where the geometry of a vibrating structure or an acoustic space is complicated and where lumped element or modal approaches cannot be used, then it is necessary to use numerical approaches. In the late 1960's with the advent of powerful digital computers, the finite element method (FEM) became feasible. Finite element approaches were first used to analyze static and then dynamic (vibration) structural problems. ${ }^{1}$ Later FEM was used with acoustics problems. In this approach the fluid is divided into a number of small fluid elements (usually rectangular or triangular) and the equations of motion are solved for the elements, ensuring that the sound pressure and volume velocity are continuous at the node points where the elements are joined. The FEM has been widely used to study the acoustical performance of automobile cabins, muffler systems and aircraft problems. ${ }^{2-6}$ Figures 1, 2 and 3 show the transmission loss (TL) of a reverse flow muffler predicted by one of the first FEM programmes designed by the author and his colleagues in the early 1970 's. Such FEM programmes are now widely available from commercial software companies. The TL of mufflers comprised of combinations of side in-centre out 